\title{
A One-Way Proof-of-Work Protocol to Protect Controllers in Software-Defined Networks
}

\author{
Jingrui Li and Tilman Wolf \\ Department of Electrical and Computer Engineering \\ University of Massachusetts, Amherst, MA, USA \\ \{jingrui,wolf\}@umass.edu
}

\begin{abstract}
Connection setup in software-defined networks (SDN) requires considerable amounts of processing, communication, and memory resources. Attackers can target SDN controllers with simple attacks to cause denial of service. We proposed a defense mechanism based on a proof-of-work protocol. The key characteristics of this protocol, namely its one-way operation, its requirement for freshness in proofs of work, its adjustable difficulty, its ability to work with multiple network providers, and its use of existing TCP/IP header fields, ensure that this approach can be used in practice.
\end{abstract}

\section{Categories and Subject Descriptors}

C.2.0 [Computer-Communication Networks]: GeneralSecurity and protection

\section{Keywords}

security, denial-of-service, attack, defense, Internet

\section{INTRODUCTION}

Software-defined networks (SDN) separate data plane operations implemented by simple lookups in network switches, and control plane operations implemented in SDN controllers $[1,3]$. SDN switches match incoming traffic against a set of flow rules that have been installed by the controller. For new connections that have no matching rules, the switch forwards the flow information to the SDN controller. The controller then makes a routing decision and informs all switches along the path so that they can install a matching rule in their flow tables.

The basic operation of SDN exhibits an imbalance between the small amount of work that is necessary to trigger large amounts of work that is then performed by the SDN controller. An attacker can exploit this imbalance by simply sending traffic with random 5 -tuples, triggering a route computation with each packet and effectively overloading the SDN controller and filling flow tables in switches.

Permission to make digital or hard copies of part or all of this work for personal or classroom use is granted without fee provided that copies are not made or distributed for profit or commercial advantage and that copies bear this notice and the full citation on the first page. Copyrights for third-party components of this work must be honored. For all other uses, contact the owner/author(s).

ANCS '16 March 17-18, 2016, Santa Clara, CA, USA

(c) 2016 Copyright held by the owner/author(s).

ACM ISBN 978-1-4503-4183-7/16/03

DOI: http://dx.doi.org/10.1145/2881025.2889481
To level this imbalance, we introduce the Controller Protection Protocol (CPP), which requires systems wanting to connect through an SDN network to commit resources before an SDN controller commits resources for route computation and setup. In our case, the connecting system needs to include a proof-of-work (POW) [2] with the initial packet of a connection. The SDN controller can verify the correctness of the POW easily and thus discard attack traffic with invalid POWs with low overhead. Using this approach, an attacker needs to dedicate a large amount of computational resources in order to send large amounts of attack traffic that triggers route computation on the SDN controller, thus making an attack potentially prohibitively expensive.

\section{PROOF-OF-WORK SDN PROTOCOL}

The main idea for the Controller Protection Protocol is to use a proof of work during connection setup. This proof of work requires the end-system requesting the connection to commit considerable resources before resources are committed on the side of the SDN controller. When an attacker sends large numbers of connection requests (without committing the resources to include valid proofs of work in each packet), then these packets can be identified and discarded with very little overhead.

The connection setup process based on the Controller Protection Protocol is shown in Figure 1. In CPP, the endsystem first computes a proof of work. The result from this computation, i.e., the POW, is included in the first packet sent by the new connection (e.g., the TCP SYN). When the first SDN switch encounters the packet from this new connection, it forwards the connection information, including the POW, to the SDN controller. The controller then checks the validity of POW before performing path computation or any other actions. If the validation fails, the controller discards the packet and the connection is not set up (i.e., no path computation takes place and no forwarding rule is installed in the SDN switches), as shown in Figure 2. If the validation succeeds, the path computation and forwarding rule installation is performed as in conventional SDN. Once the connection has been established, later packets of that connection do not contain a POW, but are forwarded by the SDN switches as in conventional SDN.

\section{ONE-WAY OPERATION}

Since the proof of work needs to be calculated by the endsystem initiating the connection before it is known which path the packet takes through the Internet and which network providers are encountered, we need to design a proof of 


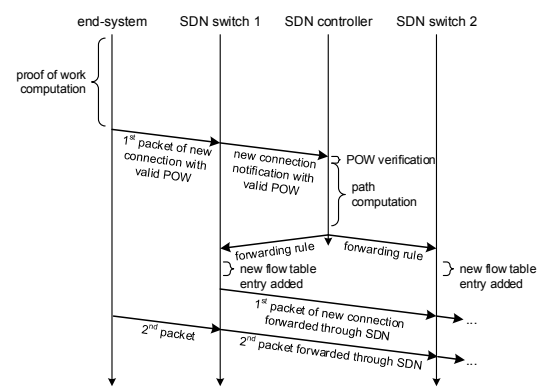

Figure 2: Denial-of-service attack

Figure 1: Connection setup in a on software-defined network con- Figure 3: Controller Protection Protocol Authorsoftware-defined network using Con- troller using Controller Protection ity distributes current CPP parameters to SDN troller Protection Protocol. Protocol. controllers.

work that is acceptable to all network providers. We introduce a Controller Protection Protocol Authority (CPPA), which creates and distributes global CPP parameters. The resulting system architecture for the Controller Protection Protocol is shown in Figure 3. The parameter $r$ is a random number used in the proof-of-work calculation, and the parameter $c$ indicates the difficulty of generating a valid POW. These parameters are distributed to all CPP-enabled components, i.e., end-systems and SDN controllers. The parameter set $(r, c)$ needs to change over time to ensure freshness of proofs and to adapt complexity.

\section{PROOF-OF-WORK DESIGN}

In CPP, we use the proof of work (e.g., such as in bitcoin mining [4]) that can be verified with a single cryptographic hash computation. We require to find an input to a cryptographic hash computation that generates an output starting with a predetermined number of zeros. Since cryptographic hash functions are one-way functions, the entity generating the proof of work has to try by "brute force" to find a suitable input. This search process is on average time consuming and thus requires dedication of computational resources (i.e., "work"). In contrast, the verifier only needs to do a single computations to see if an input (i.e., "proof") yields an output starting with the required number of zeros.

Figure 4 shows the experimental processing time for generation and verification of a POW. The generation time is significantly larger than the verification time. Thus, the proof of work does cause the necessary commitment of resources on the side of the end-system. Also, incorrect proofs of work can be detected quickly. The ratio between generation and verification time is summarized for some parameter values in Table 1. These results show that, depending on the choice of complexity parameter, the resource commitment on the end-system can be a few hundred times the cost of verifying the proof of work or many million times.

The characteristics of the proof of work discussed above is that it is inherently pseudo-random. Therefore, it is possible to utilize existing header fields, i.e., IP identification field and TCP sequence number, that use randomized values to carry the proof of work. Thus, we believe CPP presents an effective approach to protecting SDN from DoS attacks.

\section{Acknowledgments}

This material is based upon work supported by the National Science Foundation under Grant No. 1421448.

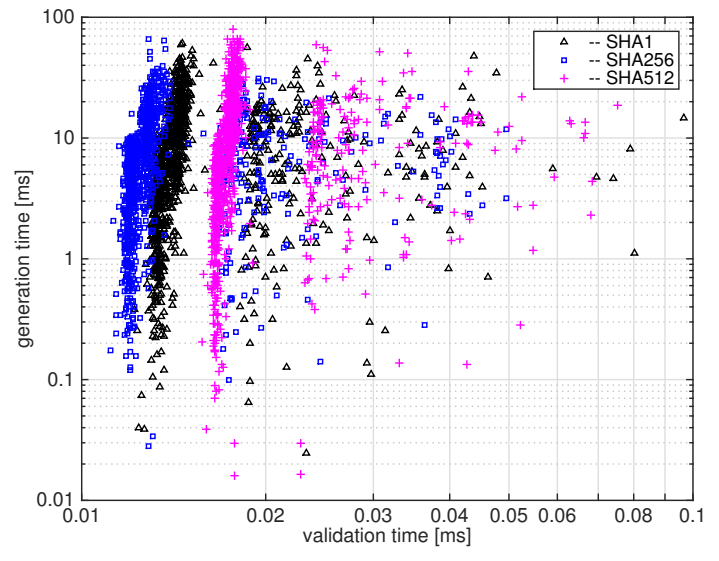

Figure 4: Distribution of generation and verification times for proofs of work in CPP (ten zeroes).

Table 1: Average ratio of generation time and verification time of proofs of work for different cryptographic functions and required number of zeros.

\begin{tabular}{|l|r|r|r|r|r|}
\hline hash & \multicolumn{5}{|c|}{ required number of zeros } \\
function & 8 & 10 & 12 & 16 & 24 \\
\hline SHA-1 & 134 & 575 & $2.23 \mathrm{k}$ & $35.7 \mathrm{k}$ & $6.37 \mathrm{M}$ \\
\hline SHA-256 & 136 & 581 & $2.16 \mathrm{k}$ & $35.7 \mathrm{k}$ & $7.86 \mathrm{M}$ \\
\hline SHA-512 & 159 & 646 & $2.75 \mathrm{k}$ & $42.0 \mathrm{k}$ & $8.64 \mathrm{M}$ \\
\hline
\end{tabular}

\section{REFERENCES}

[1] M. Casado, M. J. Freedman, J. Pettit, J. Luo, N. McKeown, and S. Shenker. Ethane: taking control of the enterprise. In SIGCOMM '07: Proceedings of the 2007 conference on Applications, technologies, architectures, and protocols for computer communications, pages 1-12, Kyoto, Japan, Aug. 2007.

[2] C. Dwork and M. Naor. Pricing via processing or combatting junk mail. In E. F. Brickell, editor, Proc. of Advances in Cryptology (CRYPTO), volume 740 of Lecture Notes in Computer Science, pages 139-147. Springer, 1993.

[3] N. McKeown, T. Anderson, H. Balakrishnan, G. Parulkar, L. Peterson, J. Rexford, S. Shenker, and J. Turner. OpenFlow: enabling innovation in campus networks. SIGCOMM Computer Communication Review, 38(2):69-74, Apr. 2008.

[4] S. Nakamoto. Bitcoin: A peer-to-peer electronic cash system. http://bitcoin.org/bitcoin.pdf, 2008. 